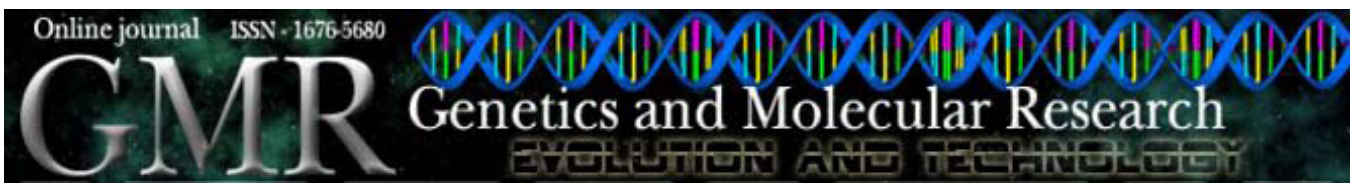

\title{
Prognostic value of TP53 Pro47Ser and Arg72Pro single nucleotide polymorphisms and the susceptibility to gliomas in individuals from Southeast Brazil
}

\author{
G.R. Pinto ${ }^{1,2}$, F.K.N. Yoshioka ${ }^{1}$, R.L.L. Silva ${ }^{2}$, C.A. Clara ${ }^{3}$, M.J. Santos ${ }^{3}$, \\ J.R.W. Almeida ${ }^{3}$, R.R. Burbano ${ }^{4}$, J.A. Rey $^{5}$ and C. Casartelli ${ }^{2}$ \\ ${ }^{1}$ Laboratório de Genética Humana e Biologia Molecular, \\ Universidade Federal do Piauí, Parnaíba, PI, Brasil \\ ${ }^{2}$ Laboratório de Oncogenética, Departamento de Genética, \\ Faculdade de Medicina de Ribeirão Preto, Universidade de São Paulo, \\ Ribeirão Preto, SP, Brasil \\ ${ }^{3}$ Fundação Pio XII, Hospital de Câncer de Barretos, Barretos, SP, Brasil \\ ${ }^{4}$ Laboratório de Citogenética Humana e Genética Toxicológica, \\ Departamento de Biologia, Universidade Federal do Pará, Belém, PA, Brasil \\ ${ }^{5}$ Laboratorio de Oncogenética Molecular, \\ Departamento de Cirugía Experimental, Hospital Universitario La Paz, \\ Madrid, Spain \\ Corresponding author: G.R. Pinto \\ E-mail: pintogr@gmail.com
}

Genet. Mol. Res. 7 (1): 207-216 (2008)

Received November 14, 2007

Accepted January 2, 2008

Published February 26, 2008

\begin{abstract}
The TP53 tumor suppressor gene codifies a protein responsible for preventing cells with genetic damage from growing and dividing by blocking cell growth or apoptosis pathways. A common single nucleotide polymorphism (SNP) in TP53 codon 72 (Arg72Pro) induces a 15-fold decrease of apoptosis-inducing ability and has been associated with susceptibility to human cancers. Recently, another TP53 SNP at codon 47 (Pro47Ser) was reported to have a low apoptosis-
\end{abstract}


inducing ability; however, there are no association studies between this SNP and cancer. Aiming to study the role of TP53 Pro47Ser and Arg72Pro on glioma susceptibility and oncologic prognosis of patients, we investigated the genotype distribution of these SNPs in 94 gliomas (81 astrocytomas, 8 ependymomas and 5 oligodendrogliomas) and in 100 healthy subjects by the polymerase chain reaction-restriction fragment length polymorphism approach. Chi-square and Fisher exact test comparisons for genotype distributions and allele frequencies did not reveal any significant difference between patients and control groups. Overall and disease-free survivals were calculated by the Kaplan-Meier method, and the log-rank test was used for comparisons, but no significant statistical difference was observed between the two groups. Our data suggest that TP53 Pro47Ser and Arg72Pro SNPs are not involved either in susceptibility to developing gliomas or in patient survival, at least in the Brazilian population.

Key words: Gliomas; Single nucleotide polymorphisms; TP53; Pro47Ser; Arg72Pro

\section{INTRODUCTION}

The TP53 gene, located at 17p13.1, is responsible for the transcription of a site-specific DNA-binding protein and acts as a transcription factor of cell growth-regulator genes (Soussi and May, 1996). A frequency of TP53 mutations of around 50\% observed in human tumors points to complexity of antiproliferative pathways under the control of TP53 protein and demonstrates that it plays an important role in tumorigenesis (Bonafe et al., 2002). Wild-type TP53 protein prevents cells with genetic damage from growing and dividing by two distinct pathways associated with cell cycle block and apoptosis-inducing activity. This dichotomy leads to an appropriate biological response to the DNA damage: genomic integrity is reestablished by DNA repair and the transient cell cycle blockade is lifted, or the cell undergoes apoptosis if DNA damage persists (Lane, 1992; Wang et al., 1995).

A critical site in the TP53 protein for apoptosis-signaling is a proline-rich region located between codon 64 and 92. At codon 72, in exon 4, a frequent functional single nucleotide polymorphism (SNP) that leads to an arginine-proline amino acid change (Arg72Pro) has been reported. Dumont et al. (2003) reported that the Arg72 allele, if in homozygosis, has an apoptosis-inducing ability 15-fold higher than does the Pro72 allele. According to Leu et al. (2004), this high apoptosis-inducing ability of the Arg72 allele is in part due to its mitochondrial location which makes it possible for TP53 to have a direct interaction with pro-apoptotic BAK protein. Studies on this SNP function were the basis for testing its impact on the risk and progression of tumors, where the less apoptotic allele Pro 72 was associated with increased risk for development of tumors (Granja et al., 2004; Hishida et al., 2004; Xi et al., 2004; IgnaszakSzczepaniak et al., 2006; Perrone et al., 2007; Toyama et al., 2007).

Recently, another SNP that induces a proline-serine change in exon 4 at codon 47 of TP53 gene (Pro47Ser) has also demonstrated a significant decrease in TP53 protein apoptosisinducing ability (Li et al., 2005). A critical event in TP53-inducing apoptosis ability is phos- 
phorylation of the serine residue at codon 46, where allele Pro47 acts as a substrate of prolinedirected kinases, for example MAPK1 protein. Li et al. (2005) reported that allele Ser47, a poor substrate for MAPK1, has an apoptosis-inducing ability 5-fold lower than does the wild Pro47 allele. According to our present knowledge, there are no studies that show whether an association exists between TP53 Pro47Ser SNP and tumoral processes.

In the present research, we conducted a case-control study and examined the genotype distribution of TP53 Pro47Ser and Arg72Pro SNPs, using the polymerase chain reaction-restriction fragment length polymorphism (PCR-RFLP) approach, to further evaluate its possible relevance in susceptibility to gliomas and in determining the oncologic prognosis of patients.

\section{MATERIAL AND METHODS}

\section{Study population}

A total of 94 gliomas were analyzed, which had been surgically resected from previously untreated patients under the care of the Neurosurgery Department of Fundação Pio XII, Cancer Hospital of Barretos (Barretos, SP, Brazil). Tumor types and stages were determined according to WHO criteria (Kleihues et al., 2002) by two experienced pathologists. The clinical outcome, including length of survival, was obtained from patient records and by contacting each patient's general practitioner. The mean follow-up period for all patients was 47.62 weeks (range $=0.12-118.14$ ). Blood samples of 100 healthy individuals were collected for controls. Because of the highly heterogeneous ethnic composition of the Brazilian population, the individuals of the control group were selected from the general population of São Paulo State, with no family history of cancer in first-degree relatives. The control sample was matched for gender and mean age with the patient group. The mean age of both patient and control groups was 45 years old, and $69.14 \%$ of patients and $69 \%$ of controls were 40 years old or older. The collection and use of tumor and blood samples for this study were previously approved by the appropriate institutional Ethics Committee.

\section{DNA extraction and primer construction}

DNA extraction was performed using proteinase $\mathrm{K}$ and phenol-chloroform according to routine molecular biology protocols. Primers were constructed using the Gene Runner 3.05 program (Hasting Software, Inc.) from gene sequence of the TP53 Pro47Ser and Arg72Pro polymorphisms, obtained in the dbSNP of NCBI (Accession numbers: rs1800371 and rs1042522, respectively). Table 1 shows the primers and PCR product sizes.

Table 1. Polymerase chain reaction (PCR) primers for the amplification of TP53 Pro47Ser and Arg72Pro single
nucleotide polymorphisms.
\begin{tabular}{lcccc}
\hline Gene & Primer & Sequence (5'-3') & Length (bp) & PCR product (bp) \\
\hline TP53 & Pro47Ser-F & CTG GTA AGG ACA AGG GTT GG & 20 & 201 or \\
& Pro47Ser-R & TCA TCT GGA CCT GGG TCT TC & 20 & $185^{*}$ \\
& Arg72Pro-F & GAA GAC CCA GGT CCA GAT GA & 20 & 152 \\
& Arg72Pro-R & CTG CCC TGG TAG GTT TTC TG & 20 & \\
\hline
\end{tabular}

*Size divergence due to a 16-bp in/del intronic polymorphism near the TP53 Pro47Ser single nucleotide polymorphism. 


\section{Polymerase chain reaction-restriction fragment length polymorphism and sequencing}

PCR was carried out in a final volume of $25 \mu \mathrm{L}$ containing $50 \mathrm{ng}$ genomic DNA template, 1X PCR buffer (Biotools) with $2 \mathrm{mM} \mathrm{MgCl}, 0.4 \mu \mathrm{M}$ of each primer (Invitrogen), $50 \mu \mathrm{M}$ dNTPs (Amersham Biosciences), and 0.5 U DNA polymerase (Biotools). For PCR amplification, the standard program was used as follows: one initial denaturation step at $94^{\circ} \mathrm{C}$ for $5 \mathrm{~min}$, followed by 35 denaturation cycles of $30 \mathrm{~s}$ at $94^{\circ} \mathrm{C}, 30 \mathrm{~s}$ of annealing at $54^{\circ} \mathrm{C}$, and $30 \mathrm{~s}$ of extension at $72^{\circ} \mathrm{C}$, followed by a final elongation cycle at $72^{\circ} \mathrm{C}$ for $5 \mathrm{~min}$.

For RFLP, the PCR products of TP53 Pro47Ser and Arg72Pro SNPs were digested with $M s p I\left(4 \mathrm{U}\right.$ at $37^{\circ} \mathrm{C}$ for $4 \mathrm{~h}$ ) and $B s t \mathrm{UI}\left(2 \mathrm{U}\right.$ at $60^{\circ} \mathrm{C}$ for $4 \mathrm{~h}$ ) (New England BioLabs), respectively. MspI recognizes a restriction site at Pro47 allele ( $\left.\mathrm{C}^{\boldsymbol{\nabla}} \mathrm{CGG}\right)$ and generates two fragments of different sizes (156 or $140 \mathrm{bp}$ and $45 \mathrm{bp}$ ), while Ser47 allele generates only one fragment of 201 or $185 \mathrm{bp}$ (size divergences due to a $16 \mathrm{bp}$ in/del intronic polymorphism near the TP53 SNP, as shown in Table 1). In the same way, BstUI generates two fragments of different sizes (52 and $100 \mathrm{bp}$ ) by recognizing a restriction site at $\operatorname{Arg} 72$ allele $\left(\mathrm{CG}^{\mathbf{\nabla}} \mathrm{CG}\right)$, while Pro72 allele generates only one (152 bp). DNA fragments were electrophoresed through a $10 \%$ acrylamide:bisacrylamide gel (19:1), and then stained with silver nitrate.

The genotypes of $>10 \%$ of the samples were reassessed to confirm the results. Also, selected PCR products were purified and submitted to bidirectional sequencing to further confirm the authenticity of genotype analysis. PCR products were purified with ExoSAP (USB), followed by sequencing with DYEnamic ET Dye Terminator Kit (Amersham Biosciences), according to the manufacturer's specifications. Sequencing reactions were performed on MegaBACE 1000 (GE Healthcare).

\section{Statistical analysis}

The chi-square test was used to verify whether genotype distributions were in HardyWeinberg equilibrium. Observed frequencies of genotypes in gliomas were compared to controls using chi-square or Fisher exact tests when expected frequencies were small. KaplanMeier curves were constructed to assess overall survival and disease-free survival rates, and differences among groups were analyzed by the log-rank test. Statistical significance was set at $\mathrm{P}<0.05$. Statistical analyses were performed with S-Plus 2000 (Insightful, Inc.) and GraphPad Prism 4.0 (GraphPad Software, Inc.) softwares.

\section{RESULTS}

A total of 94 glioma patients and 100 control subjects were included in this study. The patients comprised 59 males and 35 females $(\mathrm{M} / \mathrm{F}$ ratio $=1.69)$ and the control subjects consisted of 63 males and 37 females $(\mathrm{M} / \mathrm{F}$ ratio $=1.7)$. Mean age in patient and control groups was 45 years (range $=1-75$ and 18-72, respectively). No significant gender- or age-related differences were observed between the groups $(\mathrm{P}>0.05)$. Eighty-one patients had astrocytomas (11 grade I, 23 grade II, 8 grade III, and 39 grade IV glioblastomas), 5 oligodendrogliomas ( 3 grade II and 2 grade III), and 8 ependymomas ( 5 grade II and 3 grade III). Forty-two tumors of 94 gliomas were classified as low grade (grades I and II) and 52 as high grade (grades III and IV). No significant difference in stratification by tumor grade was observed among the groups (TP53 Pro47Ser and Arg72Pro, $\mathrm{P}=$ 0.585 and $\mathrm{P}=0.104$, respectively). 
Genotype frequencies in controls and patients were in Hardy-Weinberg equilibrium. Allele and genotype frequencies of TP53 Pro47Ser and Arg72Pro SNPs in controls and patients were not significantly different. As shown in Table 2, for TP53 Pro47Ser SNP, Ser47 allele frequency in controls was 0.01 , while in patients it was $0.02(\mathrm{P}=1.000)$. The frequencies of Pro/Pro and Pro/Ser genotypes among controls were 98 and $2 \%$, while in patients these were 96.8 and $3.2 \%$, respectively $(\mathrm{P}=0.675)$. For TP53 Arg72Pro SNP, Pro72 allele frequency in controls was 0.31 , while in patients it was $0.26(\mathrm{P}=0.531)$. Frequencies of $\mathrm{Arg} / \mathrm{Arg}$, Arg/Pro, and Pro/Pro genotypes among controls were 48,42 and $10 \%$, while in patients these were $56.4,36.2$ and $7.4 \%$, respectively $(\mathrm{P}=0.488)$.

\begin{tabular}{|c|c|c|c|c|c|c|}
\hline & & \multicolumn{2}{|c|}{ Controls } & \multicolumn{2}{|c|}{ Patients } & \multirow[t]{2}{*}{$\mathrm{P}^{*}$} \\
\hline & & $\mathrm{N}$ & $\%$ & $\mathrm{~N}$ & $\%$ & \\
\hline \multirow[t]{3}{*}{ TP53 Pro47Ser } & Pro/Pro & 98 & $(98 \%)$ & 91 & $(96.8 \%)$ & 0.675 \\
\hline & Pro/Ser & 2 & $(2 \%)$ & 3 & $(3.2 \%)$ & \\
\hline & Ser47 allele frequency & & 0.01 & & 0.02 & 1.000 \\
\hline \multirow[t]{4}{*}{ TP53 Arg72Pro } & $\operatorname{Arg} / \operatorname{Arg}$ & 48 & $(48 \%)$ & 53 & $(56.4 \%)$ & 0.488 \\
\hline & $\mathrm{Arg} /$ Pro & 42 & $(42 \%)$ & 34 & $(36.2 \%)$ & \\
\hline & Pro/Pro & 10 & $(10 \%)$ & 7 & $(7.4 \%)$ & \\
\hline & Pro72 allele frequency & & 0.31 & & 0.26 & 0.531 \\
\hline
\end{tabular}

Data are reported as number with percent in parentheses. *P values were obtained using the Fisher exact test or the chi-square test.

The mean follow-up period for all patients was 47.62 weeks (range $=0.12-118.14$ ). For 53 patients who survived the follow-up period (censored patients), mean follow-up was 62.07 weeks. For 41 patients who died during the follow-up period, mean follow-up time was 28.93 weeks. Survival curves for overall survival and disease-free survival in the patients were not significantly different for any of the TP53 SNPs tested (Kaplan-Meier analysis; P > 0.05) (Figures 1-4).

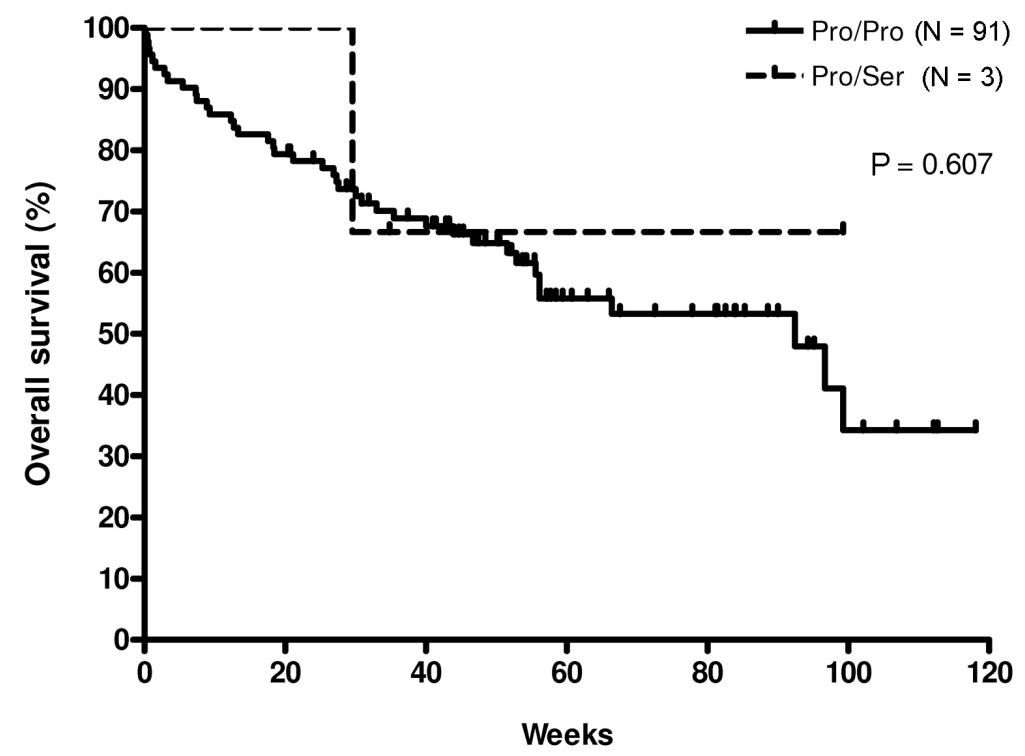

Figure 1. Overall survival in patients according to TP53 Pro47Ser single nucleotide polymorphism. 


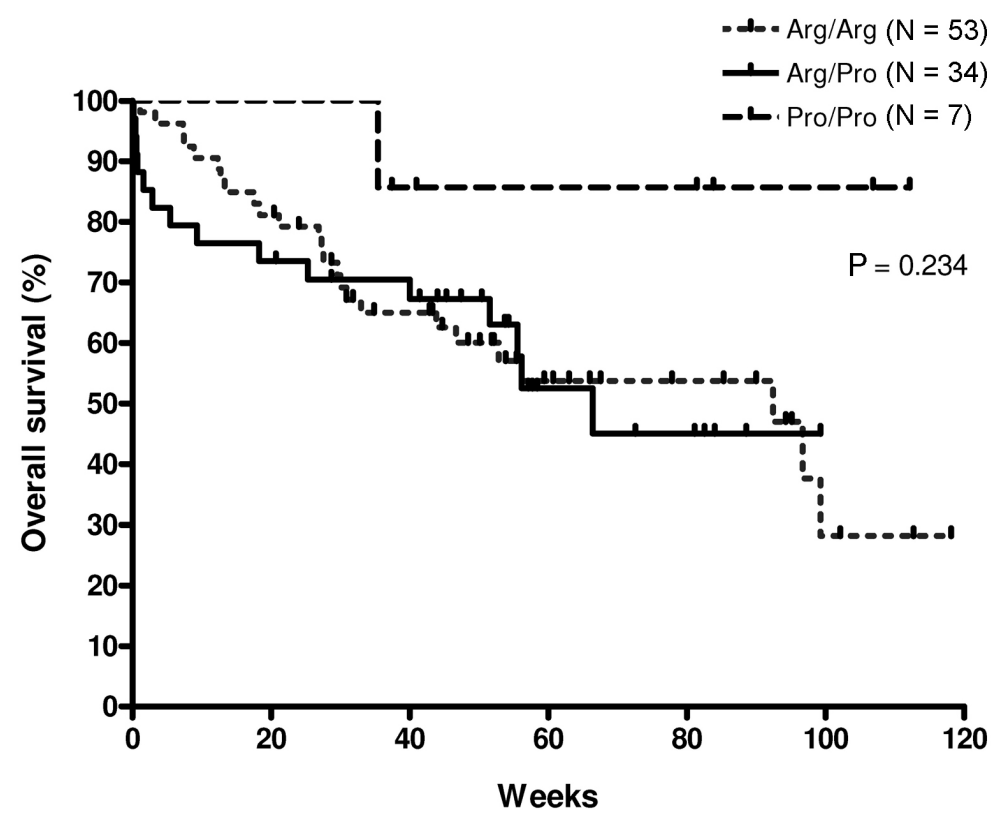

Figure 2. Overall survival in patients according to TP53 Arg72Pro single nucleotide polymorphism.

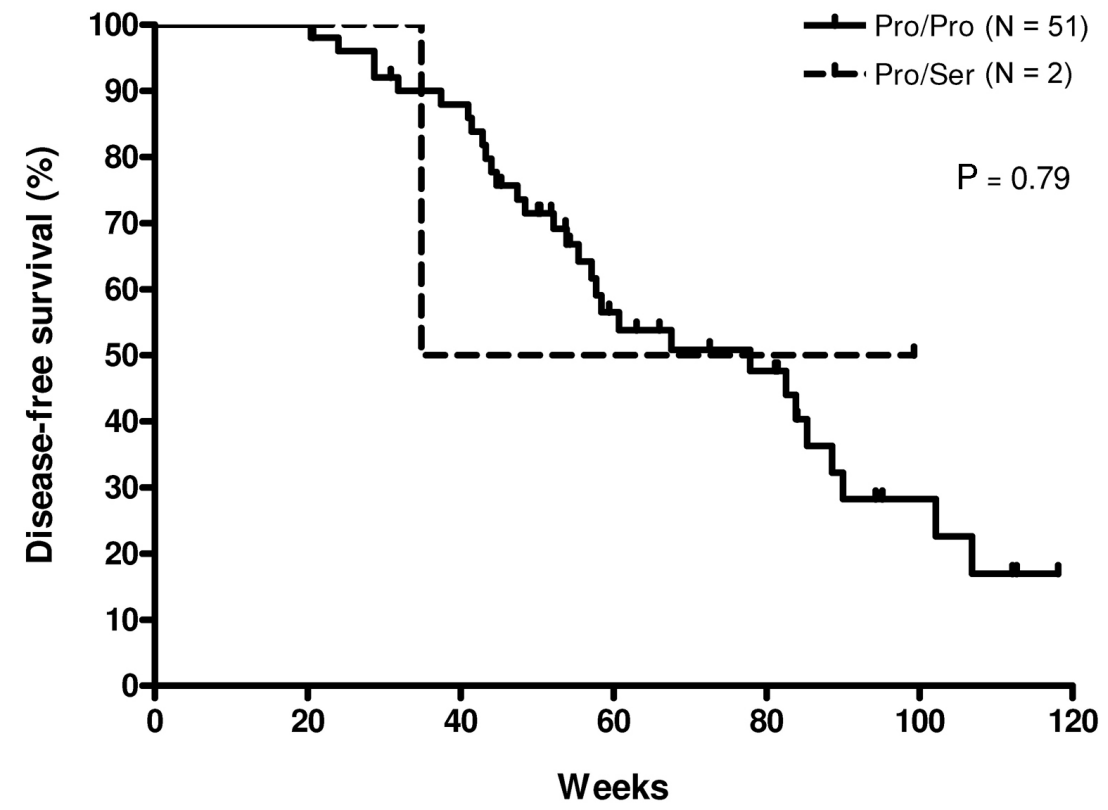

Figure 3. Disease-free survival in patients according to TP53 Pro47Ser single nucleotide polymorphism. 


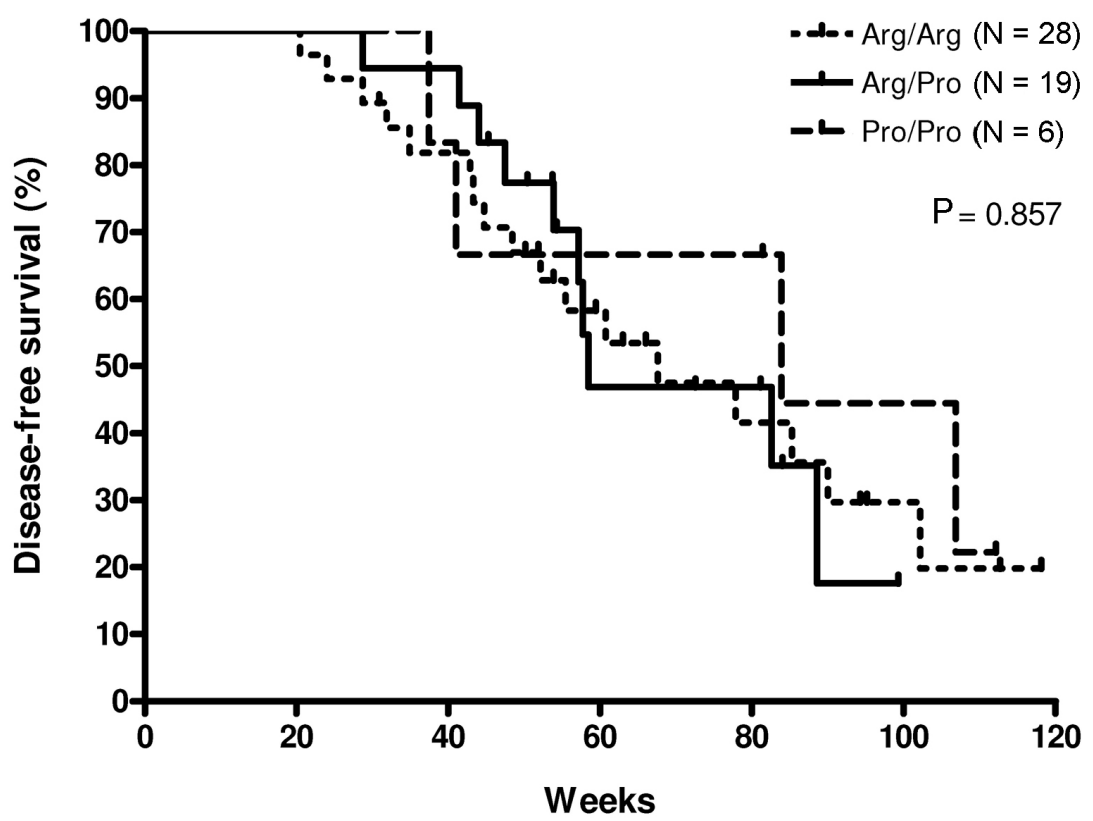

Figure 4. Disease-free survival in patients according to TP53 Arg72Pro single nucleotide polymorphism.

\section{DISCUSSION}

SNPs are the most abundant variations in the human genome and have become increasingly popular in the genetic study of several diseases, due to their quick, inexpensive and accurate analysis. The identification of SNPs as risk factors for different cancer types can be important for prevention, diagnosis and prognosis (Kirk et al., 2002). For this reason, the major aim of this case-control study was to investigate the relationship between TP53 Pro47Ser and Arg72Pro SNPs and susceptibility to glioma and patient survival.

Published research substantially lacks information on TP53 Pro47Ser SNP. FelleyBosco et al. (1993) were the first to report about the Ser47 allele prevalence in Afro-American patients, showing a frequency less than 0.05 . However, they did not find this allele in any of the 69 Caucasians from their cohort. Our results show a frequency of 0.01 for the Ser47 allele in the control group and 0.02 in patients, similar to a recent survey in 200 Afro-American individuals who showed a Ser47 allele frequency of 0.01 (Li et al., 2005).

As far we know, this is the first study that investigates the association between TP53 Pro47Ser SNP and cancer susceptibility and oncologic prognosis of patients, more specifically with regard to glioma. Our data showed that neither glioma susceptibility $(\mathrm{P}=0.675)$ nor patient survival (overall and disease-free survival, $\mathrm{P}=0.607$ and 0.79 , respectively) was associated with variant alleles.

Several studies have investigated association between TP53 Arg72Pro SNP and an increased risk for developing tumors and results have shown that this SNP is a risk factor for adrenocortical, colorectal, breast, lung, head and neck, and cervical-uterine cancers, among 
many others (Papadakis et al., 2002; Shen et al., 2002; Buyru et al., 2003; Sifuentes and Reyes, 2003; Ignaszak-Szczepaniak et al., 2006; Zhu et al., 2007). However, none of the investigations have shown strong evidence, and this association remains unclear (Drummond et al., 2002; Oren, 2003; Khadang et al., 2007). With regard to Arg72Pro alleles, Arg72 in homozygosis has been reported as a risk factor for cervical cancer (Qie et al., 2002) while the Pro72 allele in homozygosis has been reported as a high risk factor for pulmonary, hepatic and nasopharyngeal cancers (Wang et al., 1995; Yu et al., 1999; Tsai et al., 2002). Genotype Arg/Pro has been associated with an increased susceptibility to tobacco-induced lung adenocarcinoma (Fan et al., 2000). In our study, we did not observe an association between TP53 Arg72Pro SNP and susceptibility to glioma $(\mathrm{P}=0.488)$.

The heterogeneity of association of TP53 Arg72Pro SNP is at least in part due to its large ethnic and geographical variation (Zehbe et al., 1998). The Pro72 allele, for example, has shown a north-south frequency gradient of 0.17 in Sweden to 0.63 in Nigeria (Beckman et al., 1994). In Western Europe (France, Switzerland, and Norway), the United States, Central and South America (Mexico, Costa Rica and Peru), and Japan, Arg72 allele is the most frequent, ranging from 0.60 to 0.83 . However, frequencies higher than 0.40 for Pro72 allele were reported in Afro-Americans and Chinese (Weston et al., 1992; Jin et al., 1995; Ngan et al., 1999; Peixoto et al., 2001). In our study, we identified a frequency of 0.69 for $\operatorname{Arg} 72$ allele in the control group and 0.74 in patients, results similar to other studies conducted in Brazil. Granja et al. (2004), besides demonstrating an association between the Pro/Pro genotype and thyroid cancer, also reported a frequency of 0.65 for Arg 72 allele in a control group from southeast Brazil. Khayat et al. (2005), in a case-control study of gastric adenocarcinoma in the State of Pará, northern Brazil, described a frequency of 0.69 for Arg 72 allele in their control group.

The TP53 Arg72Pro SNP role in cancer patient prognosis is still more conflicting. While several studies have revealed that TP53 Arg72Pro SNP has no significant effect on oncologic prognosis for patients with pancreatic, testicular and prostate tumors (Wu et al., 1995; Dong et al., 2003), other studies have confirmed this association for patients with breast and lung tumors (Wang et al., 1995; Toyama et al., 2007). In our study, we were not able to demonstrate an association between TP53 Arg72Pro SNP and glioma patient survival (overall and disease-free survival, $\mathrm{P}=0.234$ and 0.857 , respectively).

Jones et al. (2004) reported that homozygote individuals for Pro72 allele from nonpolyposis colorectal cancer-predisposed families have disease manifestation 13 years earlier than homozygote individuals for Arg72 allele. However, unlike Jones et al. (2004), we did not observe an association between TP53 Arg72Pro alleles and age of glioma patients ( $\mathrm{P}>0.05)$.

There are few studies on the association between TP53 Arg72Pro SNP and gliomas. Biros et al. (2002) and Uno et al. (2006) did not show any association of this SNP in astrocytoma samples. Furthermore, Parhar et al. (2005) have suggested a possible association between TP53 Arg72Pro SNP and susceptibility to brain tumors, particularly high-grade astrocytomas. Our results corroborate only the studies of Biros et al. (2002) and Uno et al. (2006), since we were not able to demonstrate any association between TP53 Arg72Pro SNP and tumor grade $(\mathrm{P}=0.104)$, as demonstrated by Parhar et al. (2005).

We conclude that there is no association between TP53 Pro47Ser and Arg72Pro genotypes and glioma susceptibility or patient survival, at least in the Brazilian population. Nevertheless, future studies on larger populations from other parts of the world are essential for a definitive conclusion about these results. 


\section{ACKNOWLEDGMENTS}

We are grateful to the patients who took part in this investigation. We thank Márcio Rogério Penha and Vanderci Massaro de Oliveira for the technical support provided in this research. Research supported by Conselho Nacional de Desenvolvimento Científico e Tecnológico (CNPq), Fundação de Amparo à Pesquisa do Estado de São Paulo (FAPESP), Fundação de Apoio ao Ensino, Pesquisa e Assistência do Hospital das Clínicas da Faculdade de Medicina de Ribeirão Preto (FAPEAM), and Financiadora de Estudos e Projetos (FINEP CT-INFRA/FADESP) (Grant number: 1017-01). R.R. Burbano was the recipient of a PQ-2 fellowship (number 308256/2006-9) from CNPq.

\section{REFERENCES}

Beckman G, Birgander R, Sjalander A, Saha N, et al. (1994). Is p53 polymorphism maintained by natural selection? Hum. Hered. 44: 266-270.

Biros E, Kalina I, Kohut A, Bogyiova E, et al. (2002). Allelic and haplotype frequencies of the p53 polymorphisms in brain tumor patients. Physiol. Res. 51: 59-64.

Bonafe M, Barbi C, Storci G, Salvioli S, et al. (2002). What studies on human longevity tell us about the risk for cancer in the oldest old: data and hypotheses on the genetics and immunology of centenarians. Exp. Gerontol. 37: 1263-1271.

Buyru N, Tigli H and Dalay N (2003). P53 codon 72 polymorphism in breast cancer. Oncol. Rep. 10: 711-714.

Dong M, Nio Y, Yamasawa K, Toga T, et al. (2003). p53 alteration is not an independent prognostic indicator, but affects the efficacy of adjuvant chemotherapy in human pancreatic cancer. J. Surg. Oncol. 82: 111-120.

Drummond SN, De Marco L, Pordeus IA, Barbosa AA, et al. (2002). TP53 codon 72 polymorphism in oral squamous cell carcinoma. Anticancer Res. 22: 3379-3381.

Dumont P, Leu JI, Della Pietra A III, George DL, et al. (2003). The codon 72 polymorphic variants of p53 have markedly different apoptotic potential. Nat. Genet. 33: 357-365.

Fan R, Wu MT, Miller D, Wain JC, et al. (2000). The p53 codon 72 polymorphism and lung cancer risk. Cancer Epidemiol. Biomarkers Prev. 9: 1037-1042.

Felley-Bosco E, Weston A, Cawley HM, Bennett WP, et al. (1993). Functional studies of a germ-line polymorphism at codon 47 within the p53 gene. Am. J. Hum. Genet. 53: 752-759.

Granja F, Morari J, Morari EC, Correa LA, et al. (2004). Proline homozygosity in codon 72 of p53 is a factor of susceptibility for thyroid cancer. Cancer Lett. 210: 151-157.

Hishida A, Matsuo K, Tajima K, Ogura M, et al. (2004). Polymorphisms of p53 Arg72Pro, p73 G4C14-to-A4T14 at exon 2 and 21 Ser31Arg and the risk of non-Hodgkin's lymphoma in Japanese. Leuk. Lymphoma 45: 957-964.

Ignaszak-Szczepaniak M, Horst-Sikorska W, Sawicka J, Kaczmarek M, et al. (2006). The TP53 codon 72 polymorphism and predisposition to adrenocortical cancer in Polish patients. Oncol. Rep. 16: 65-71.

Jin X, Wu X, Roth JA, Amos CI, et al. (1995). Higher lung cancer risk for younger African-Americans with the Pro/Pro p53 genotype. Carcinogenesis 16: 2205-2208.

Jones JS, Chi X, Gu X, Lynch PM, et al. (2004). p53 polymorphism and age of onset of hereditary nonpolyposis colorectal cancer in a Caucasian population. Clin. Cancer Res. 10: 5845-5849.

Khadang B, Fattahi MJ, Talei A, Dehaghani AS, et al. (2007). Polymorphism of TP53 codon 72 showed no association with breast cancer in Iranian women. Cancer Genet. Cytogenet. 173: 38-42.

Khayat AS, Lobo GL, Moura LE, de Assumpcao PP, et al. (2005). Polymorphisms of the TP53 codon 72 and WRN codon 1367 in individuals from Northern Brazil with gastric adenocarcinoma. Clin. Exp. Med. 5: 161-168.

Kirk BW, Feinsod M, Favis R, Kliman RM, et al. (2002). Single nucleotide polymorphism seeking long term association with complex disease. Nucleic Acids Res. 30: 3295-3311.

Kleihues P, Louis DN, Scheithauer BW, Rorke LB, et al. (2002). The WHO classification of tumors of the nervous system. J. Neuropathol. Exp. Neurol. 61: 215-225.

Lane DP (1992). Cancer. p53, guardian of the genome. Nature 358: 15-16.

Leu JI, Dumont P, Hafey M, Murphy ME, et al. (2004). Mitochondrial p53 activates Bak and causes disruption of a BakMcl1 complex. Nat. Cell Biol. 6: 443-450.

Li X, Dumont P, Della PA, Shetler C, et al. (2005). The codon 47 polymorphism in p53 is functionally significant. J. Biol. Chem. 280: 24245-24251. 
Ngan HY, Liu VW and Liu SS (1999). Risk of cervical cancer is not increased in Chinese carrying homozygous arginine at codon 72 of p53. Br. J. Cancer 80: 1828-1829.

Oren M (2003). Decision making by p53: life, death and cancer. Cell Death Differ. 10: 431-442.

Papadakis ED, Soulitzis N and Spandidos DA (2002). Association of p53 codon 72 polymorphism with advanced lung cancer: the Arg allele is preferentially retained in tumours arising in Arg/Pro germline heterozygotes. Br. J. Cancer 87: 1013-1018.

Parhar P, Ezer R, Shao Y, Allen JC, et al. (2005). Possible association of p53 codon 72 polymorphism with susceptibility to adult and pediatric high-grade astrocytomas. Brain Res. Mol. Brain Res. 137: 98-103.

Peixoto GD, Hsin LS, Snijders P, Wilmotte R, et al. (2001). Absence of association between HPV DNA, TP53 codon 72 polymorphism, and risk of oesophageal cancer in a high-risk area of China. Cancer Lett. 162: 231-235.

Perrone F, Mariani L, Pastore E, Orsenigo M, et al. (2007). p53 codon 72 polymorphisms in human papillomavirusnegative and human papillomavirus-positive squamous cell carcinomas of the oropharynx. Cancer 109: 2461-2465.

Qie M, Zhang Y and Wu J (2002). Study on the relationship between cervical cancer and p53 codon 72 polymorphism. HuaXi YiKe DaXиe XиeBao 33: 274-275.

Shen H, Zheng Y, Sturgis EM, Spitz MR, et al. (2002). P53 codon 72 polymorphism and risk of squamous cell carcinoma of the head and neck: a case-control study. Cancer Lett. 183: 123-130.

Sifuentes AA and Reyes RM (2003). Risk factors for cervico-uterine cancer associated to HPV: p53 codon 72 polymorphism in women attending hospital care. Ginecol. Obstet. Mex. 71: 12-15.

Soussi T and May P (1996). Structural aspects of the p53 protein in relation to gene evolution: a second look. J. Mol. Biol. 260: 623-637.

Toyama T, Zhang Z, Nishio M, Hamaguchi M, et al. (2007). Association of TP53 codon 72 polymorphism and the outcome of adjuvant therapy in breast cancer patients. Breast Cancer Res. 9: R34.

Tsai MH, Lin CD, Hsieh YY, Chang FC, et al. (2002). Prognostic significance of the proline form of p53 codon 72 polymorphism in nasopharyngeal carcinoma. Laryngoscope 112: 116-119.

Uno M, Oba-Shinjo SM, Wakamatsu A, Huang N, et al. (2006). Association of TP53 mutation, p53 overexpression, and p53 codon 72 polymorphism with susceptibility to apoptosis in adult patients with diffuse astrocytomas. Int. J. Biol. Markers 21: 50-57.

Wang XW, Yeh H, Schaeffer L, Roy R, et al. (1995). p53 modulation of TFIIH-associated nucleotide excision repair activity. Nat. Genet. 10: 188-195.

Weston A, Perrin LS, Forrester K, Hoover RN, et al. (1992). Allelic frequency of a p53 polymorphism in human lung cancer. Cancer Epidemiol. Biomarkers Prev. 1: 481-483.

Wu WJ, Kakehi Y, Habuchi T, Kinoshita H, et al. (1995). Allelic frequency of p53 gene codon 72 polymorphism in urologic cancers. Jpn. J. Cancer Res. 86: 730-736.

Xi YG, Ding KY, Su XL, Chen DF, et al. (2004). p53 polymorphism and p21WAF1/CIP1 haplotype in the intestinal gastric cancer and the precancerous lesions. Carcinogenesis 25: 2201-2206.

Yu MW, Yang SY, Chiu YH, Chiang YC, et al. (1999). A p53 genetic polymorphism as a modulator of hepatocellular carcinoma risk in relation to chronic liver disease, familial tendency, and cigarette smoking in hepatitis B carriers. Hepatology 29: 697-702.

Zehbe I, Voglino G, Delius H, Wilander E, et al. (1998). Risk of cervical cancer and geographical variations of human papillomavirus 16 E6 polymorphisms. Lancet 352: 1441-1442.

Zhu ZZ, Wang AZ, Jia HR, Jin XX, et al. (2007). Association of the TP53 codon 72 polymorphism with colorectal cancer in a Chinese population. Jpn. J. Clin. Oncol. 37: 385-390. 